\title{
Investigation of separate adsorbent particles under adsorption-desorption conditions with the use of infrared thermography and computer simulation
}

\author{
By B. G. Vainer*, A. B. Ayupov** and M. S. Melgunov**
}

* Rzhanov Institute of Semiconductor Physics SB RAS, Novosibirsk State University; 13 Lavrentyev av., Novosibirsk, 630090, Russia, BGV@isp.nsc.ru

** G.K.Boreskov Institute of Catalysis SB RAS, Novosibirsk State University;

5 Lavrentyev av., Novosibirsk, 630090, Russia, artem@catalysis.ru, max@catalysis.ru

Nowadays, infrared thermography (IRT) attracts attention as an efficient tool for adsorption-desorption studies of porous materials [1-4], especially, of silica gels and zeolites. These studies help to optimize the gas purification and separation processes [4], and to improve monitoring and controlling fire and explosion hazards in the industry [3]. The fundamental problem, which should be solved here, is to obtain a further insight into the physics of processes occurring on the surface of materials with a developed inner structure, that, in turn, is important for applications to catalysis.

Until recently, experimental thermographic investigation of adsorption boiled down to obtaining the temperature profiles, which appeared and moved along or across adsorbent bed. Meanwhile, information about the real processes, which occur within a single particle, is needed to simulate properly the heat and mass transfer in such media. The aim of this work is the solution for the last problem.

The particles were placed in a glass filter funnel (figure 1a), in which the gas mixture was fed at the bottom via digital flow controller of the textural characteristics analyzer "Termosorb" (Russia). The applied technology has allowed us to quantify the adsorption-desorption characteristics of separate particles of different solid adsorbents. Spatial (angular) resolution of a standard lens of an infrared camera permits to detect the temperature variation in an extended adsorbent bed, but usually it is not designed to study a single particle or separate particles in the bed. For this functionality, we made a special laboratory setup, which was equipped with an additional quartz lens and permitted to resolve separate particles (figure $1 \mathrm{~b}$ ).

Figure 2 shows typical thermograms of the particles in the adsorption mode (left) and desorption mode (right). Here are shown some control points at which the temperature was measured for the quantitative description of the processes. The checkpoints indicated near pellets were used in high-sensitive regimes to compensate an excessive background level associated with the reflection of infrared radiation from the structural.

It is found that the adsorption and desorption processes on the particles occur in different ways depending on the flow velocity of the adsorbate and the particle size. The comparative experimental examples for the adsorption characteristics for values of flow of the water vapor-nitrogen mixture of $1 \mathrm{ml} / \mathrm{s}$ and $0.5 \mathrm{ml} / \mathrm{s}$ are shown in figure 3 . In particular, the particle size reduction at low-flow experiments results in the shift of the temperature curve maximum to shorter times.

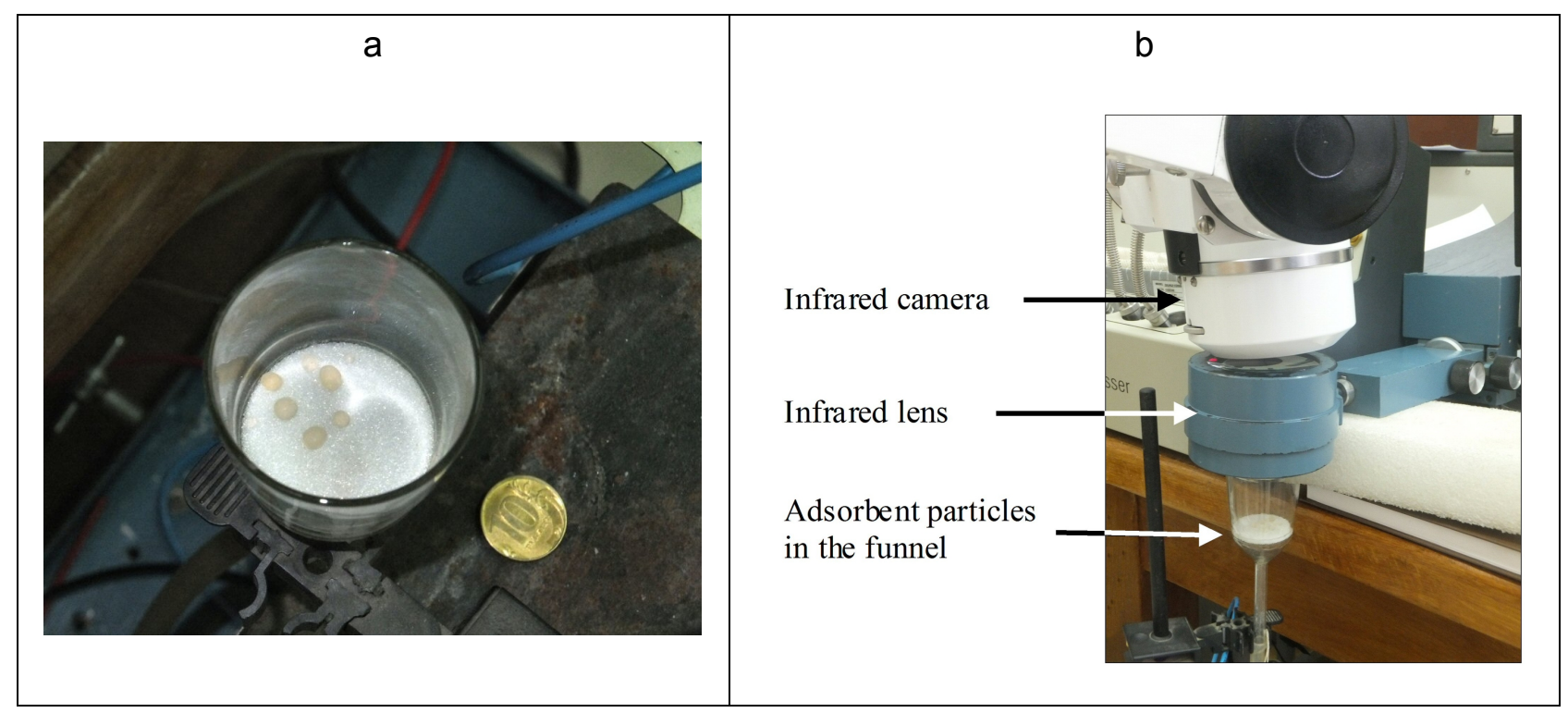

Fig. 1. a - Grains of silica, placed in the funnel for making IRT studies; $b$ - design of optical part of measurement system. 


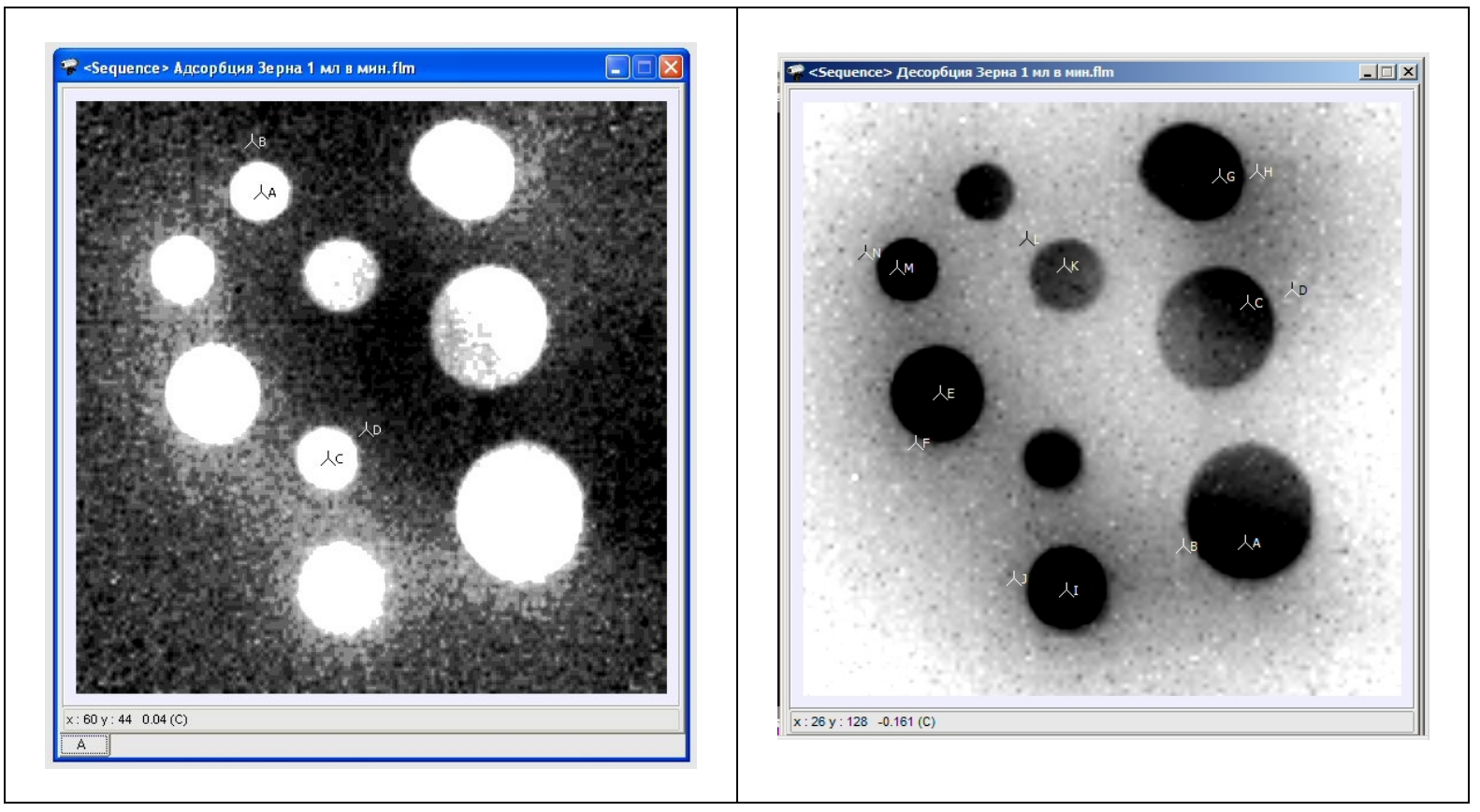

Fig. 2. Thermograms of separate particles of adsorbent (silica gel) in the adsorption (left) and desorption (right) modes.

The mathematical model, which describes the thermal processes on separate particles of the adsorbent, has been elaborated, and a good agreement is obtained between the experimental data and the simulation results (figure 4). It is based on the model of adsorption in the pellet of porous material in the non-isothermal conditions described in [5]. This model includes the following equations:

$$
\begin{gathered}
G_{1}(y, \theta) \frac{\partial y}{\partial \tau}+G_{2}(y, \theta) \frac{\partial \theta}{\partial \tau}=\frac{1}{\eta^{S}} \frac{\partial}{\partial \eta}\left(\eta^{S} H(y, \theta) \frac{\partial y}{\partial \eta}\right) \\
\frac{\partial \theta}{\partial \tau}=\left.\beta \frac{C_{0}}{C_{\mu 0}}(1+S) H(y, \theta) \frac{\partial y}{\partial \eta}\right|_{\eta=1}-\operatorname{LeBi}\left(\theta-\theta_{b}\right) ; \\
G_{1}(y, \theta)=\varepsilon+(1-\varepsilon) \frac{\partial f(C, T)}{\partial C} ; \\
G_{2}(y, \theta)=(1-\varepsilon) \frac{T_{0}}{C_{0}} \frac{\partial f(C, T)}{\partial T} ; \\
H(y, \theta)=(1+\theta)^{\alpha}+\delta_{0} \varphi(\theta) \frac{f(C, T)}{C_{\mu 0} y} \\
\varphi(\theta)=e^{\gamma_{\mu}\left(\frac{\theta}{1+\theta}\right)} .
\end{gathered}
$$

Boundary and initial conditions in dimensionless coordinates look like

$$
\begin{gathered}
\eta=0 \Leftrightarrow \frac{\partial y}{\partial \eta}=0 ; \eta=1 \Leftrightarrow-\left.H(y, \theta) \frac{\partial y}{\partial \eta}\right|_{\eta=1}=B i\left(\left.y\right|_{\eta=1}-y_{b}\right) ; \\
\tau=0 \Leftrightarrow y=y_{i} ; \theta=\theta_{i}
\end{gathered}
$$

The parameters and constants in eqs. (1)-(8) are as follows: 
1. The dimensionless time and space coordinates $\tau=\frac{\varepsilon D_{p 0} t}{R^{2}} ; \eta=\frac{r}{R}$;

2. Dimensionless concentration and temperature $y=\frac{C}{C_{0}} ; x=\frac{C_{\mu}}{C_{\mu 0}} ; \theta=\frac{T-T_{0}}{T_{0}} ;$

3. Ratio of surface diffusion to the bulk one, and the Biot number $\delta_{0}=\frac{(1-\varepsilon) D_{\mu 0}^{0} C_{\mu 0}}{\varepsilon D_{p 0} C_{0}} ; B i=\frac{k_{m} R}{\varepsilon D_{p 0}}$;

4. Dimensionless activation energy for diffusion and heat capacity $\gamma_{\mu}=\frac{E_{\mu}}{R_{g} T_{0}} ; \beta=\frac{Q C_{\mu 0}}{\left\langle\rho C_{p}\right\rangle T_{0}}$;

5. Lewis-Biot number LeBi $=\frac{a_{H} h_{f} R^{2}}{\varepsilon D_{p 0}\left\langle\rho C_{p}\right\rangle}$.

Here

$\left\langle\rho C_{p}\right\rangle$ is the averaged over the volume specific heat of pellets, $\mathrm{J} \cdot \mathrm{m}^{-3}$;

$a_{H}$ is the specific heat transfer surface, $\mathrm{m}^{-1}$;

$h_{f}$ is the thermal conductivity, $\mathrm{J} \cdot \mathrm{m}^{-2} \cdot \mathrm{s}^{-1} \cdot \mathrm{K}^{-1}$;

$T_{b}$ is the temperature of the gas surrounding the pellet, $\mathrm{K}$;

$D_{\mu 0}^{0}$ is the diffusion coefficient of the adsorbate in the adsorption layer (surface diffusion coefficient) at the reference temperature, $\mathrm{m}^{2} \cdot \mathrm{s}^{-1}$

$R_{g}$ is the molar gas constant, $8,3142 \mathrm{~J} \cdot \mathrm{mol}^{-1} \cdot \mathrm{K}^{-1}$;

$E_{\mu}$ is the molar activation energy of surface diffusion, $\mathrm{J} \cdot \mathrm{mol}^{-1}$.

$C$ is the concentration in the void volume of the adsorptive granule, $\mathrm{mol} \cdot \mathrm{m}^{-3}$ (void volume);

$\varepsilon$ is the granule porosity (open porosity), $\mathrm{m}^{3} \mathrm{~m}^{-3}$;

$C_{\mu}$ is the concentration of adsorbate in the adsorption layer, $\mathrm{mol} \cdot \mathrm{m}^{-3}$ (adsorption layer);

$t$ is the time, s;

$r$ is the space coordinate, $\mathrm{m}$;

$S$ is the shape factor of the pellet ( 0 - slab, $1-$ cylinder, 2 - sphere).

Thermal conductivity $h_{f}$ was calculated using the empirical relation presented in [6].

The model presented here allows you to set the properties of the adsorbate and the properties of the adsorber. Adsorbate properties are defined by determining the density, viscosity and thermal conductivity constants. Adsorbateadsorbent interaction is set by determining the heat of adsorption and adsorption equilibrium constants. The parameters of the adsorbent granules are defined by determining the radius, and the constants of the Knudsen diffusion surface, as well as by setting the activation energy for surface diffusion. The parameters of the adsorber are defined by determining the flow rate of the carrier gas and the concentration of adsorbate, and the temperature of the carrier gas.

The system of equations was solved numerically using the MATLAB assisted with the method of orthogonal collocation. The solutions obtained were compared to the experimental curves of granule temperature on the time. From comparison of the fitted plots, it is clear that the simulation allows one to characterize the whole thermogram obtained. The model parameters for the investigated granules are shown in Table. 1.

As can be seen from Table. 1, the LeBi, reflecting the rate of heat exchange with the environment, has a value in the range of $0.5-9$, which substantially exceeds the expected one for the investigated flow rates (for Reynolds numbers less than one, this coefficient should be less than 0.1). The observed discrepancy could be explained by a significant 
influence on the temperature distribution of the thermal conductivity within the contact between granule and adsorber (funnel). Indeed, using infrared thermography we fixed heating the reactor bottom around the granules, which should not be, if there is no such thermal contact.

The adsorption equilibrium parameters are selected from our previous studies of the test sample KSK-1. We were unable to perform a satisfactory simulation of the observed infrared curves based on the initial conditions under which the adsorbent granule was completely free of the adsorbate before gas puffing. It turned out that this assumption must be a satisfactory description of a certain amount of the adsorbate in the investigated pellets.

During the simulation we deliberately fixed constant adsorption isotherm parameters, as it was found that random selection of heat and mass transfer parameters can justify any amount of the adsorption equilibrium constant and its limit. It is, therefore, evident that the thermographic studies have a really quantitative value only if some additional precise measurements of concentration, the size of objects and the flow velocity of the adsorbate are made.

Infrared thermography has shown itself as a sensitive and demonstrative tool to study adsorption. Thus, this method is of direct obtaining experimental data that would otherwise be obtained only in indirect measurements. The combination of infrared thermography with numerical processing methods and mathematical modeling seems a powerful tool for verifying the physical behavior of adsorption systems.

An extremely important feature of this method is its visibility, which makes it indispensable in finding and justifying assumptions for mathematical modeling (uniform or not heat distribution within the granules, the possible mechanisms of heat transfer, etc.). However, the use of infrared thermography requires the material of reactors and adsorbers to be transparent in the infrared range, which imposes restrictions on the range of studied process conditions. Infrared thermography also requires calibration procedures and verification for the calculation of objects actual temperature, which is necessary for accurate calculation of physicochemical constants of adsorbents and adsorbates. Despite these shortcomings, infrared thermography is a promising experimental method for the study of adsorption and related diffusion processes.

Table. 1. Parameters of the model (1)-(8) fitted for experimental thermograms.

\begin{tabular}{|c|c|c|c|c|c|c|c|c|}
\hline $\begin{array}{c}\text { Check } \\
\text { point }\end{array}$ & $\begin{array}{c}\text { Grain } \\
\text { radius } \\
\mathrm{R}, \\
\mathrm{mm}\end{array}$ & $\begin{array}{c}\text { Initial } \\
\text { pressure of } \\
\text { adsorbtive } \\
\mathrm{p}_{\mathrm{i}} \\
\mathrm{kPa}\end{array}$ & $\begin{array}{c}\text { Bulk } \\
\text { pressure of } \\
\text { adsorbtive } \\
\mathrm{p}_{\mathrm{b}} \\
\mathrm{kPa}\end{array}$ & $\begin{array}{c}\text { Langmuir } \\
\text { constant } \\
\mathrm{b}_{0} \\
1 / \mathrm{kPa}\end{array}$ & $\begin{array}{c}\text { Initial } \\
\text { concentration } \\
\text { of adsorbate } \\
\mathrm{C}_{\mu} \\
\mathrm{mol} / \mathrm{cm}^{3}\end{array}$ & $\begin{array}{c}\text { Activation } \\
\text { energy of } \\
\text { surface } \\
\text { diffusion } \\
E_{\mu} \\
\mathrm{J} / \mathrm{mol}\end{array}$ & $\begin{array}{c}\text { Heat of } \\
\text { adsorption } \\
\text { Q, } \\
\mathrm{J} / \mathrm{mol}\end{array}$ & $\begin{array}{l}\text { Lewis- } \\
\text { Biot } \\
\text { number } \\
\text { LeBi }\end{array}$ \\
\hline \multicolumn{9}{|c|}{ Adsorption, flow rate $1 \mathrm{ml} / \mathrm{s}(0.628 \mathrm{~mm} / \mathrm{s})$} \\
\hline$A$ & 2.5 & 1 & 3.331 & 0.000215 & 0.02 & 10000 & 45000 & 5 \\
\hline C & 2.2 & 1.8 & 3.331 & 0.000215 & 0.02 & 10000 & 45000 & 4.5 \\
\hline$E$ & 1.8 & 0.8 & 3.331 & 0.000215 & 0.02 & 10000 & 45000 & 2.5 \\
\hline G & 2.0 & 0 & 3.331 & 0.000215 & 0.02 & 10000 & 45000 & 9 \\
\hline I & 1.5 & 0.5 & 3.331 & 0.000215 & 0.02 & 10000 & 45000 & 3 \\
\hline $\mathrm{K}$ & 1.4 & 1.5 & 3.331 & 0.000215 & 0.02 & 10000 & 45000 & 5 \\
\hline$M$ & 1.3 & 0.9 & 3.331 & 0.000215 & 0.02 & 10000 & 45000 & 2 \\
\hline $\mathrm{A} 2$ & 1.2 & 1.6 & 3.331 & 0.000215 & 0.02 & 10000 & 45000 & 0.8 \\
\hline $\mathrm{C} 2$ & 1.2 & 1.6 & 3.331 & 0.000215 & 0.02 & 10000 & 45000 & 0.8 \\
\hline \multicolumn{9}{|c|}{ Adsorption, flow rate $0.5 \mathrm{ml} / \mathrm{s}(0.314 \mathrm{~mm} / \mathrm{s})$} \\
\hline$A$ & 2.5 & 2.8 & 3.331 & 0.000215 & 0.02 & 10000 & 45000 & 1 \\
\hline C & 2.2 & 2.8 & 3.331 & 0.000215 & 0.02 & 10000 & 45000 & 1 \\
\hline$E$ & 1.8 & 2.6 & 3.331 & 0.000215 & 0.02 & 10000 & 45000 & 1.2 \\
\hline G & 2.0 & 2.6 & 3.331 & 0.000215 & 0.02 & 10000 & 45000 & 1.2 \\
\hline I & 1.5 & 2.5 & 3.331 & 0.000215 & 0.02 & 10000 & 45000 & 1.2 \\
\hline $\mathrm{K}$ & 1.4 & 2.85 & 3.331 & 0.000215 & 0.02 & 10000 & 45000 & 2 \\
\hline M & 1.3 & 2.7 & 3.331 & 0.000215 & 0.02 & 10000 & 45000 & 1.5 \\
\hline $\mathrm{A} 2$ & 1.2 & 0 & 3.331 & 0.000215 & 0.02 & 10000 & 45000 & 0.5 \\
\hline $\mathrm{C} 2$ & 1.2 & 0 & 3.331 & 0.000215 & 0.02 & 10000 & 45000 & 0.5 \\
\hline
\end{tabular}


It is experimentally demonstrated in this paper that FPA-based IRT enables the temperature to be found at any point of the separate adsorbent grain. The experimental technique described above makes more detailed observations of the dynamics of adsorption/desorption processes developing in the adsorptive pellets possible and represents quantitatively the characteristics of heat exchange with the environment in the course of these thermal processes.

This work was supported by the Russian Foundation for Basic Research, grant No. 11-03-00900-a, and partially by the Siberian Branch of the Russian Academy of Sciences (Integrative Project No. 40-B-2012).

\section{REFERENCES}

[1] Vainer B. G. "Quantitative characterization of vapour adsorption on solid surfaces and estimation of emissivity of solids using narrow-band short-wave infrared thermography". QIRT Journal, vol. 5, pp. 175-193, 2008.

[2] Vainer B. G., Melgunov M. S., Ayupov A. B., Fenelonov V. B. "Real-time thermal imaging and quantitative characterization of adsorption-desorption processes in zeolites and silica gels". E-Book Proceedings of the $11^{\text {th }}$ International Conference on Quantitative InfraRed Thermography, QIRT 2012, 11-14 June, 2012, Naples-Italy. Italy: University of Naples, ID-252, 9 pp., 2012.

[3] Le Cloirec P., Pre P., Delage F., Giraudet S. "Visualization of the exothermal VOC adsorption in a fixed-bed activated carbon adsorber". Environmental Techn., vol. 33, pp. 285-290, 2012.

[4] Mel'gunov M. S., Ayupov A. B., Fenelonov V. B., Vainer B. G. "Direct contact-free real-time acquisition of temperature profiles in adsorbent bed during vacuum swing adsorption". Adsorption, vol. 19, pp. 835-840, 2013.

[5] Do D. D. "Adsorption analysis: equilibria and kinetics". Vol. 2., London: Imperial College Press, 892 pp., 1998.

[6] Wakao N., Kaguei S. "Heat and mass transfer in Packed Beds". New York: Gordon and Breach Science Publishers, 1982.

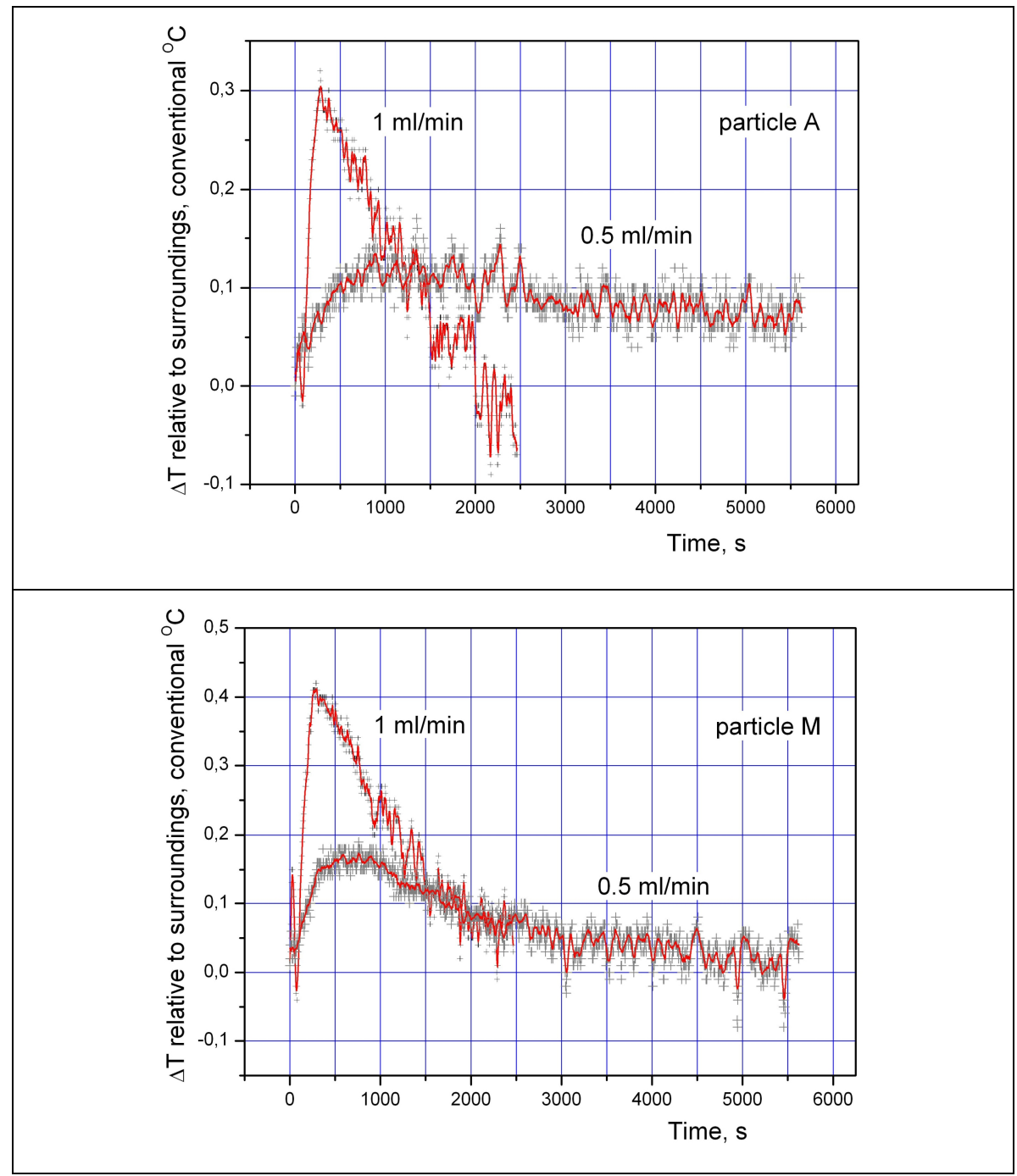

Fig. 3. The comparative experimental temporal characteristics showing the separate adsorbent particle change in temperature. Heat release is caused by the adsorption of water molecules trapped from the wet nitrogen flow. Particle size: $5.0 \mathrm{~mm}$ (on top) and $2.6 \mathrm{~mm}$ (at the bottom). 


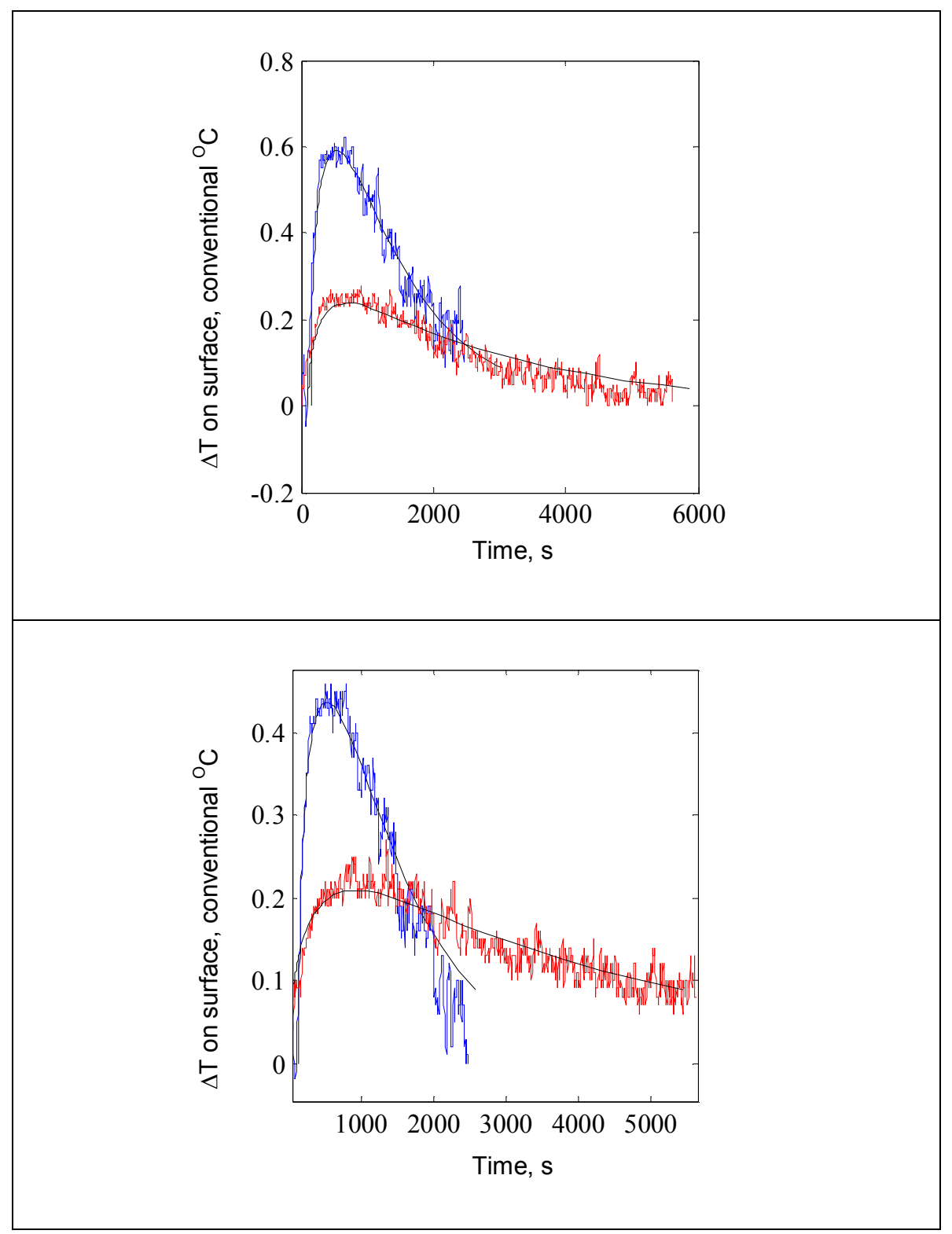

Fig. 4. Time profiles of heating of the adsorbent particles resulted from water adsorption on silica KSK-1: a correlation between experimental data (color) and simulation (black).

Particle size: $3.0 \mathrm{~mm}$ (on top) and $5.0 \mathrm{~mm}$ (at the bottom).

The initial temperature: $299 \mathrm{~K}$, flow velocity of $\mathrm{N}_{2}: 1 \mathrm{ml} / \mathrm{s}$ (blue), $0.5 \mathrm{ml} / \mathrm{s}$ (red). 